\title{
A study of a turbulent boundary layer in stalled-airfoil-type flow conditions
}

\author{
Yvan Maciel · Karl-Stéphane Rossignol · \\ Jean Lemay
}

Published online: 24 August 2006

(C) Springer-Verlag 2006

\section{Erratum to: Exp Fluids (2006) DOI 10.1007/s00348-006-0182-1}

In the original publication of this article there are some typographical errors in mathematical expressions.

We apologize for these mistakes. The correct equations are:

$\delta \propto U_{\mathrm{e}}^{-1 / \Lambda}$
Two paragraphs later (last sentence): $U_{\mathrm{zs}}=U_{\mathrm{e}} \delta^{* / \delta}$

$\frac{\mathrm{d} \delta / \mathrm{d} x}{\delta} \frac{U_{\mathrm{e}}}{\mathrm{d} U_{\mathrm{e}} / \mathrm{d} x}=-\frac{1}{\Lambda}=$ constant or $\mathrm{d} \delta / \mathrm{d} x=$ constant

$\Pi_{\mathrm{zs}}=\beta_{\mathrm{zs}} / \gamma_{\mathrm{zs}}$

The online version of the original article can be found at http://dx.doi.org//10.1007/s00348-006-0182-1.

Y. Maciel $(\bowtie) \cdot$ K.-S. Rossignol · J. Lemay

Mechanical Engineering Department, Laval University,

Quebec City, QC, Canada G1K 7P4

e-mail: ymaciel@gmc.ulaval.ca

URL: http://www.gmc.ulaval.ca 